Robert A. Hegele • D. Dan Ramdath - Matthew R. Ban

Michael N. Carruthers - Christine V. Carrington

Henian Cao

\title{
Polymorphisms in PNLIP, encoding pancreatic lipase, and associations with metabolic traits
}

Received: January 18, 2001 / Accepted: February 19, 2001

\begin{abstract}
Pancreatic lipase (EC 3.1.1.3) is an exocrine secretion that hydrolyzes dietary triglycerides in the small intestine. We developed genomic amplification primers to sequence the 13 exons of PNLIP, which encodes pancreatic lipase, in order to screen for possible mutations in cell lines of four children with pancreatic lipase deficiency (OMIM 246600). We found no missense or nonsense mutations in these samples, but we found three silent single-nucleotide polymorphisms (SNPs), namely, 96A/C in exon 3, 486C/T in exon 6 , and $1359 \mathrm{C} / \mathrm{T}$ in exon 13 . In 50 normolipidemic Caucasians, the PNLIP 96C and 486T alleles had frequencies of 0.083 and 0.150 , respectively. The PNLIP $1359 \mathrm{~T}$ allele was absent from Caucasian, Chinese, South Asian, and North American aboriginal samples, but had a frequency of 0.085 in an African sample, suggesting that it is a population-specific variant. In an association analysis of 185 African neonates, the PNLIP 1359C/T SNP genotype was significantly associated with concentrations of plasma lipoproteins. These associations were most likely due to linkage disequilibrium with another functional variant at or near PNLIP. Thus, we report three new SNPs for the PNLIP, which may serve as markers for association analyses and for pharmacogenetic studies of pancreatic lipase inhibitors.
\end{abstract}

Key words Lipolysis · Lipids - Ethnicity - Complex traits · DNA sequencing $\cdot$ Mutation $\cdot$ Metabolism

R.A. Hegele $(\bowtie) \cdot$ M.R. Ban · M.N. Carruthers $\cdot$ H. Cao Blackburn Cardiovascular Genetics Laboratory, John P. Robarts Research Institute, 406-100 Perth Drive, London, Ontario, Canada N6A $5 \mathrm{~K} 8$

Tel. +1-519-663-3461; Fax +1-519-663-3789

e-mail: robert.hegele@rri.on.ca

D.D. Ramdath · C.V. Carrington

Department of Biochemistry, Faculty of Medical Sciences, University of the West Indies, St. Augustine, Trinidad and Tobago

\section{Introduction}

Pancreatic lipase (PL; EC 3.1.1.3), a 56-kD protein, is involved in the intestinal hydrolysis of dietary triglyceride to fatty acids. PL deficiency (OMIM 246600) is associated with malabsorption of long-chain triglyceride fatty acids, failure to thrive in infancy and childhood, and absence of PL secretion upon secretin stimulation (Sheldon 1964; Rey et al. 1966). PNLIP on chromosome 10q26.1 (Davis et al. 1991) is a 13-exon gene that spans more than $20 \mathrm{~kb}$ (Sims et al. 1993) and encodes the 465-amino acid PL mature protein (Lowe et al. 1989). To date, neither PNLIP mutations in PL deficiency nor common PNLIP single-nucleotide polymorphisms (SNPs) have been reported. We thus sequenced PNLIP from the genomic DNA of subjects with PL deficiency and of several normal subjects in order to discover rare mutations and/or common SNPs.

\section{Methods}

Study subjects

Cell lines of PL-deficient probands. Lymphoblasts from four PL deficiency patients were purchased from Coriell (Camden, NJ, USA). Subject GM13278 was a 3-year-old Caucasian female with chronic diarrhea, failure to thrive, and complete deficiency of PL activity with secretin stimulation. Subject GM13322 was a 2-year-old Hispanic male with failure to thrive, developmental abnormalities, and complete deficiency of PL activity with secretin stimulation. Subject GM13343 was a 5-year-old Caucasian male with chronic diarrhea, failure to thrive, and complete deficiency of PL activity with secretin stimulation. Subject GM13344 was a 2-year-old Caucasian male with failure to thrive, developmental abnormalities, and complete deficiency of PL activity with secretin stimulation.

Trinidadian African neonates. We assessed 300 consecutive newborn infants from the Mount Hope Women's Hospital 
in Trinidad. All newborns were weighed immediately after birth. Cord blood samples were collected from each newborn. Ethnicity was determined as described (Busch et al. 1999), leaving 185 subjects of African ancestry for study. After birth, samples were taken by syringe from the umbilical vein. Ten $\mathrm{ml}$ of cord blood was transferred to ethylenediaminetetraacetic acid (EDTA)-containing tubes and was immediately centrifuged at $1700 \mathrm{~g}$ at $4{ }^{\circ} \mathrm{C}$ for $10 \mathrm{~min}$. Plasma was collected and stored for determination of plasma lipoproteins, and buffy coats were saved for extraction of genomic DNA. Each mother gave her informed consent. The protocol was approved by the Ethics Committee of the Faculty of Medical Sciences, University of the West Indies (St. Augustine).

Normal controls. Samples from a DNA archive of 50 clinically normal Caucasian subjects were analyzed to determine SNP allele frequencies. For the PNLIP 1359C/T SNP, additional samples from a DNA archive - 100 clinically normal subjects, of Chinese, East Indian, North American aboriginal, and African ethnic background - were studied to determine allele and genotype frequencies.

\section{Cell culture}

Lymphoblasts were cultured in minimal essential medium (Life Technologies, Rockville, MD, USA) supplemented with $20 \%$ heat-inactivated fetal bovine serum at $37^{\circ} \mathrm{C}$ in an atmosphere of $5 \% \mathrm{CO}_{2}$ and $95 \%$ air.

\section{Biochemical studies}

Plasma lipoproteins and apolipoproteins from neonatal samples were determined as described (Hegele et al. 1999; Brouwer et al. 2000).
Genomic DNA isolation

Genomic DNA from cultured lymphoblasts was obtained using a commercial method, following the manufacturer's instructions (Puregene, Gentra Systems, Minneapolis, MN, USA). Genomic DNA from neonatal samples had been prepared as described (Busch et al. 1999; Hegele et al. 1999).

Screening PNLIP for mutations

In order to amplify PNLIP coding regions and intron-exon boundaries from genomic DNA, a primer set was developed, using GeneBank sequences (nos. L11242, L24502, L24522, L24523, L24524, L24525, L24526, L24527, L24528, and L24529). Primer sequences are shown in Table 1. Primers were each designed to anneal at $57^{\circ} \mathrm{C}$, which allowed for use of a single amplification apparatus for all reactions. Exons 1 and 2, as well as exons 7 and 8, were amplified by a single primer pair. Amplification conditions were: $94^{\circ} \mathrm{C}$ for $5 \mathrm{~min}$, followed by 30 cycles comprised of $30 \mathrm{~s}$ each at $94^{\circ} \mathrm{C}$, $57^{\circ} \mathrm{C}$, and $72^{\circ} \mathrm{C}$, ending with a single 10 -min extension step at $72^{\circ} \mathrm{C}$.

\section{Genotyping of PNLIP 1359T/C SNP}

A rapid method was used to genotype the PNLIP $1359 \mathrm{~T} / \mathrm{C}$ SNP within exon 13. Genomic DNA was amplified using primers 5'-TCCTCACTGTCACACTCCAGAT-3' and 5' TAACAGCCATTGGAGGTTCTTT-3'. The amplification conditions were: $94^{\circ} \mathrm{C}$ for $5 \mathrm{~min}$, followed by 30 cycles comprised of $30 \mathrm{~s}$ each at $94^{\circ} \mathrm{C}, 57^{\circ} \mathrm{C}$, and $72^{\circ} \mathrm{C}$, ending with a single 10 -min extension step at $72^{\circ} \mathrm{C}$. The resulting fragment was 301 base pairs in length. Digestion of the 1359C allele with $A l$ wNI (or $C a i \mathrm{I}$ ) produced a single fragment of

Table 1. PNLIP genomic DNA amplification primers

\begin{tabular}{|c|c|c|}
\hline Exon(s) & Primer sequence $\left(5^{\prime}\right.$ to $\left.3^{\prime}\right)$ & Amplification product size (bp) \\
\hline \multirow[t]{2}{*}{1 and 2} & Forward: CTTTCTTTGCATGCCATTTTTC & 332 \\
\hline & Reverse: TGAAAAGTGAATCTCTCCCAGC & \\
\hline \multirow[t]{2}{*}{3} & Forward: GCAGCAGTCATTAAAAAGAGCA & 291 \\
\hline & Reverse: GTCTCGATCTCCTGACCTCGT & \\
\hline \multirow[t]{2}{*}{4} & Forward: TACTTACTGCСССТСТCCATGT & 273 \\
\hline & Reverse: CCAGTGCCTTACCGTTTGTTA & \\
\hline \multirow[t]{2}{*}{5} & Forward: GGAATTTATCCTAGTCCTCCAGTA & 261 \\
\hline & Reverse: CCATGTGTACAGGCTTTTATGC & \\
\hline \multirow[t]{2}{*}{6} & Forward: TAACGTATCCCTGTTGTTGAGC & 203 \\
\hline & Reverse: TTCACTGTTTTTCACCAACCTG & \\
\hline \multirow{2}{*}{7 and 8} & Forward: AGAACCCGTTCATCCCTTTC & 522 \\
\hline & Reverse: TGAGCCTGTACAATTCCAAAGA & \\
\hline \multirow[t]{2}{*}{9} & Forward: GACTTTGTTCTGTGACCTGCAT & 322 \\
\hline & Reverse: TGTATAGGAAAGCACACCAGTCA & \\
\hline \multirow[t]{2}{*}{10} & Forward: CCTCATTCATCATTGTTTCTTTTC & 233 \\
\hline & Reverse: TGCACATTTTCAGTGGACATAA & \\
\hline \multirow[t]{2}{*}{11} & Forward: GTGTCTTTTATCTCCAAACTGACA & 222 \\
\hline & Reverse: AGCGAGACTCCGACTCAAAA & \\
\hline \multirow[t]{2}{*}{12} & Forward: CAGAAATGCATTGTAAGCTGGT & 399 \\
\hline & Reverse: ATCCCCTGTAGGAGGTTTCACT & \\
\hline \multirow[t]{2}{*}{13} & Forward: TCCTCACTGTCACACTCCAGAT & 301 \\
\hline & Reverse: TAACAGCCATTGGAGGTTCTTT & \\
\hline
\end{tabular}


Table 2. Clinical and biochemical traits in Trinidadian neonates of African descent according to PNLIP genotype

\begin{tabular}{llll}
\hline & & $1359 \mathrm{C} / 1359 \mathrm{~T}$ plus & \\
& $1359 \mathrm{C} / 1359 \mathrm{C}$ & $1359 \mathrm{~T} / 1359 \mathrm{~T}$ & Bonferroni $P$ value \\
\hline Number & 156 & 29 & \\
Gestational age (weeks) & $38.2 \pm 2.4$ & $38.4 \pm 2.2$ & $\mathrm{NS}$ \\
Birth weight (g) & $3151 \pm 510$ & $3084 \pm 458$ & $\mathrm{NS}$ \\
Total cholesterol (mM) & $1.61 \pm 0.47$ & $1.43 \pm 0.45$ & $<0.05$ \\
Triglycerides (mM) & $0.45 \pm 0.19$ & $0.37 \pm 0.12$ & NS \\
Apo AI (g/l) & $0.80 \pm 0.21$ & $0.69 \pm 0.24$ & $<0.05$ \\
Apo B (g/l) & $0.18 \pm 0.08$ & $0.15 \pm 0.09$ & $<0.05$ \\
Lp(a) (g/l) & $49.7 \pm 58.6$ & $28.0 \pm 26.7$ & $<0.05$ \\
\hline
\end{tabular}

Apo, Apolipoprotein; Lp, lipoproteins; NS, not significant

301 base pairs, whereas digestion of the $1359 \mathrm{~T}$ allele produced two fragments, with lengths of 211 and 90 base pairs. These fragments could be easily resolved after electrophoresis in $2 \%$ agarose gels.

\section{Statistical analysis}

SAS version 6.12 (SAS Institute, Cary, NC, USA) was used for all statistical analyses. We used $\chi^{2}$ analysis to test deviations of genotype frequencies from those predicted by the Hardy-Weinberg equation and to test differences in allele frequencies between groups. Regression analysis was used to determine sources of variation for plasma lipoprotein traits, and Bonferroni-adjusted pairwise comparisons were used to characterize between-genotype differences in quantitative traits, assuming a dominant model for the PNLIP 1359T allele. Log transformation was used to normalize the distribution of quantitative traits regression analysis, but, for clarity, untransformed means \pm SDs are shown in Table 2, comparing subjects by genotype. The nominal level of statistical significance for all analyses was $P<0.05$.

\section{Results}

\section{Identification of three PNLIP SNPS}

Genomic DNA from the cell lines from the four PLdeficient subjects and from five normal Caucasian controls were screened, using the amplification primers shown in Table 1 and the amplification program described above. There were no SNPs found in the PL-deficient subjects that altered the amino acid sequence. Three silent SNPs were found in the PL-deficient subjects, and these are shown in Fig. 1. PNLIP $96 \mathrm{~A} / \mathrm{C}$ in exon 3 was found in subject GM13322, who was homozygous for the 96C allele. PNLIP $486 \mathrm{C} / \mathrm{T}$ in exon 6 was found in subject GM13278, who was heterozygous for the $486 \mathrm{~T}$ allele. PNLIP $1359 \mathrm{C} / \mathrm{T}$ in exon 13 was found in subject GM13322, who was homozygous for the $1359 \mathrm{~T}$ allele. Homozygosity for $1359 \mathrm{~T}$ was confirmed, using AlwNI genotyping, in subject GM13322.
The PNLIP 96A/C and 486C/T SNPs were also found in the Caucasian controls, and direct sequencing of 100 Caucasian chromosomes indicated that the frequencies of the $96 \mathrm{C}$ and $486 \mathrm{~T}$ alleles were 0.083 and 0.150 , respectively. However, the PNLIP 1359T allele was completely absent from 100 Caucasian chromosomes.

Genotyping for PNLIP $1359 \mathrm{C} / \mathrm{T}$ was then performed in normal subjects from various ethnic groups. The $1359 \mathrm{~T}$ allele was absent from 200 Chinese, 200 East Indian, and 200 North American aboriginal chromosomes, but had a frequency of 0.085 in 200 African chromosomes. In the African sample, the frequencies were $84.3 \%$ for the $1359 \mathrm{C} / \mathrm{C}$ genotype, $15.1 \%$ for the $1359 \mathrm{C} / \mathrm{T}$ genotype, and $0.6 \%$ for the $1359 \mathrm{~T} / \mathrm{T}$ genotype. The observed genotype frequencies did not deviate significantly from those predicted by the HardyWeinberg equation. Thus, the PNLIP 1359T allele appeared to be an ethnic-specific variant, occurring only in subjects of African descent.

Genetic associations in Trinidadian neonates of African descent

In a sample of 185 African neonates, the PNLIP 1359T allele frequency was 0.081 , with no difference between males and females, and no deviation of genotype frequencies from those predicted by the Hardy-Weinberg equation (data not shown).

The association of the PNLIP $1359 \mathrm{C} / \mathrm{T}$ SNP with metabolic traits, assuming a dominant model for $1359 \mathrm{~T}$, was examined in this sample of 185 neonates of African origin, using linear regression analysis, with sex and gestational age included as covariates. The PNLIP $1359 \mathrm{C} / \mathrm{T}$ SNP genotype was a significant source of variation for plasma concentrations of total cholesterol, apolipoprotein (apo) AI, apo B and lipoprotein (Lp) (a). Sex was a significant source of variation for cholesterol and apo AI (data not shown). Gestational age was a significant determinant of birth weight in this sample, as subjects with lower gestational age also had a lower birth weight (data not shown). Pairwise comparisons (shown in Table 2) indicated that subjects with the PNLIP 1359T allele had lower plasma total cholesterol, apo $\mathrm{AI}$, apo $\mathrm{B}$, and $\mathrm{Lp}(\mathrm{a})$ compared with findings in subjects with the PNLIP $1359 \mathrm{C} / \mathrm{C}$ genotype. 
a

PL

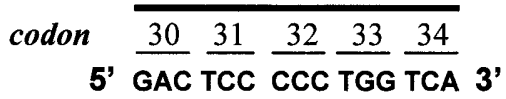

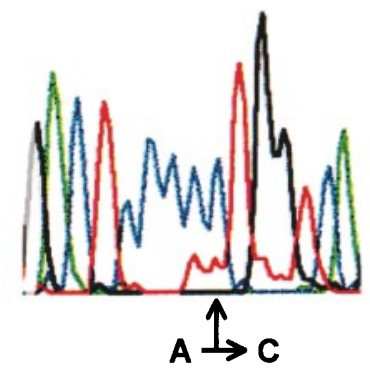

codon $30 \quad 31 \quad 32 \quad 33 \quad 34$

5' GAC TCC CCA TGG TCA 3'

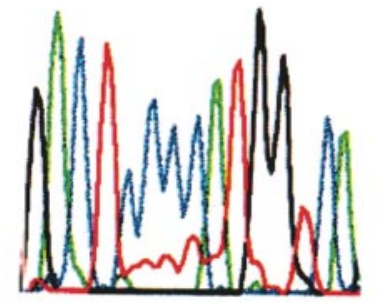

b

$P L$
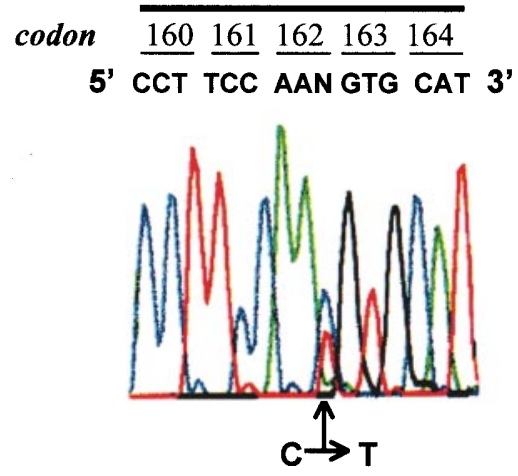

codon $160 \quad 161 \quad 162 \quad 163 \quad 164$

5' CCT TCC AAC GTG CAT 3'

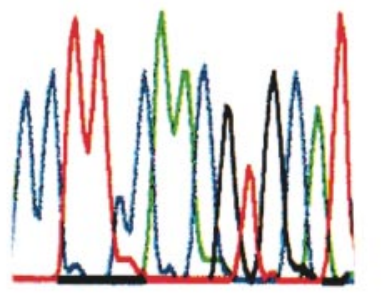

c
Fig. 1a-c. $P N L I P$ single-nucleotide polymorphisms (SNPs). Electrophoretogram tracings of sequences from genomic DNA templates in subjects screened for mutations in PNLIP. Mutant sequences are shown in each upper panel, and wild-type sequences are shown in each lower panel. Codon numbers, orientation, and nucleotide sequences are indicated. Mutant nucleotides are indicated by arrows. a Subject GM13322 is a homozygote for 96C; b subject GM13278 is a heterozygote for 486T; and c subject GM13322 is a homozygote for 1359T

\section{Discussion}

We report herein: (1) a novel set of primers that amplified the entire PNLIP coding sequence at a single annealing temperature, allowing for effective use of amplification hardware, (2) the use of these amplification primers for genomic DNA sequencing, which has resulted in: (3) the identification of three novel SNPs in PNLIP, of which one, namely, $1359 \mathrm{C} / \mathrm{T}$, is specific to people of African descent; and (4) novel associations of the PNLIP 1359C/T SNP with plasma lipoproteins in neonatal Trinidadians of African descent. There were no PNLIP mutations in subjects with PL deficiency.

Two of the novel PNLIP SNPs, namely, 96A/C and $486 \mathrm{C} / \mathrm{T}$, were shown by direct sequencing of genomic DNA to be relatively common in Caucasians. These SNPs might be useful as markers for association studies. Because these SNPs do not affect naturally occurring restriction sites, other methods, such as mismatch amplification or allelespecific hybridization, will be required to detect them. The development of such methods was beyond the scope of the present report.

The availability of PNLIP SNPs may also be helpful for pharamacogenetic studies. Pharmacological inhibition of PL is currently of wide clinical interest. For example, Xenical (orlistat; Hoffmann LaRoche, Nutley, NJ, USA) has been tested in over 17000 patients in clinical trials and has been prescribed to more than 1000000 patients worldwide (Blackburn 1999). There appears be some interindividual variation in response, both with respect to the efficacy and the tolerability of this medication (Hill et al. 1999). Having PNLIP markers may be helpful in genetically stratifying people for studies of the sources of interindividual variation in efficacy and tolerability.

The association of PNLIP 1359C/T SNP genotype with plasma lipoprotein traits in Trinidadian neonates of African descent, while of some interest, is not likely due to a functional impact of this silent SNP. Rather, this SNP may be in linkage disequilibrium with other variants in PNLIP, or another nearby gene on chromosome 10q26.1, which underlie the association. The observations are compatible with suggestions that there may be an early effect of genetic determinants on complex plasma lipoprotein traits (Low 1998). But because there is substantial evolution over time of all plasma lipoproteins when compared with baseline values at birth (van Biervliet et al. 1980), the significance of the observed associations over the long term is unclear. Furthermore, $\mathrm{PL}$ is probably not a direct determinant of any neonatal metabolic trait. PNLIP mRNA is not expressed in either 
human or rat fetal liver (Payne et al. 1994; Yang et al. 2000), and reaches physiological levels only several weeks after birth (Payne et al. 1994). This is in contrast to the PL-related proteins PNLIPRP1 (OMIM 604422) and PNLIPRP2 (OMIM 604423), whose function is unknown, but whose expression is evident early in fetal development (Giller et al. 1992; Payne et al. 1994; Yang et al. 2000), indicating that these genes have different temporal expression.

We found no coding sequence or intron-exon boundary SNPs in the cell lines from PL-deficient subjects. There are several possible explanations for this observation. First, because we did not sequence the $P N L I P$ promoter region, we cannot rule out the possibility of a disease mutation in that region in some or all PL-deficient subjects. Second, PL requires a $12-\mathrm{kD}$ polypeptide co-factor called pancreatic colipase (OMIM 120105), encoded by the CLPS gene. It is possible that the PL-deficient phenotype could result from CLPS mutations. Third, there are at least two other PNLIP-related genes, called PNLIPRP1 and PNLIPRP2 (Giller et al. 1992), whose function is unknown. It is this possible that mutations affecting the function of the PNLIPRP1 and PNLIPRP2 gene products might contribute to PL deficiency.

In summary, we have identified genomic DNA amplification primers for $P N L I P$, in addition to common SNPs, which may be useful for association and pharmacogenetic studies. The association of the PNLIP 1359C/T SNP with lipoproteins in African neonates probably does not result from a functional change. However, the association is compatible with an effect of genetic determinants on plasma lipoprotein traits, and should be confirmed in larger studies using diverse study samples.

Acknowledgments We thank Hans Mulder, Birret Fokkens, Susan Tom, Balram Mahabir, and the Mount Hope Women's Hospital nursing staff for collecting and processing the DNA samples; we also thank Pearl Campbell and Jian Wang. Supported by the Research and Publications Committee, University of the West Indies (St. Augustine), by the Commonwealth Caribbean Medical Research Council, and by operating grants from the MRC Canada (MT13430) and the Heart and Stroke Foundation of Ontario (NA3629). Dr. Hegele holds a Canada Research Chair in Human Genetics, and is a Career Investigator of the Heart and Stroke Foundation of Ontario.

\section{References}

Blackburn GL (1999) Blockade of pancreatic lipase. Am J Clin Nutr $71: 845$

Brouwer DA, Mulder H, Fokkens B, Ramsewak S, Muskiet FA, Ramdath DD (2000) Cord blood apolipoprotein-E genotype distribution and plasma lipid indices in newborns of different ethnicity. Ann Hum Biol 27:367-375

Busch CP, Ramdath DD, Ramsewak S, Hegele RA (1999) Association of PON2 variation with birth weight in Trinidadian neonates of South Asian ancestry. Pharmacogenetics 9:351-356

Davis RC, Diep A, Hunziker W, Klisak I, Mohandas T, Schotz MC, Sparkes RS, Lusis AJ (1991) Assignment of human pancreatic lipase gene $(P N L I P)$ to chromosome 10q24-q26. Genomics 11:1164-1166

Figarella C, Negri GA, Sarles H (1972) Presence of co-lipase in a congenital pancreatic lipase deficiency. Biochim Biophys Acta 280:205-210

Giller T, Buchwald P, Blum-Kaelin D, Hunziker W (1992) Two novel human pancreatic lipase related proteins, hPLRP1 and hPLRP2: differences in colipase dependence and in lipase activity. J Biol Chem 267:16509-16516

Hegele RA, Ban MR, Busch CP, Ramsewak S, Ramdath DD (1999) Lipoprotein-genotype associations in Trinidadian neonates. Clin Biochem 32:429-437

Hill JO, Hauptman J, Anderson JW, Fujioka K, O'Neil PM, Smith DK, Zavoral JH, Aronne LJ (1999) Orlistat, a lipase inhibitor, for weight maintenance after conventional dieting: a 1-year study. Am J Clin Nutr 69:1108-1116

Low PS, Saha N, Tay JS, Arulkumaran S (1998) Influence of PvuII (intron 6) polymorphism of the lipoprotein lipase gene on cord plasma lipid and apolipoprotein levels in Indian and Chinese newborns of Singapore. Pediatr Res 43:240-244

Lowe ME, Rosenblum JL, Strauss AW (1989) Cloning and characterization of human pancreatic lipase cDNA. J Biol Chem 264:2004220048

Payne RM, Sims HF, Jennens ML, Lowe ME (1994) Rat pancreatic lipase and two related proteins: enzymatic properties and mRNA expression during development. Am J Physiol 1994;266:G914-921

Rey J, Frezal J, Royer P, Lamy M (1966) L'absence congenitale de lipase pancreatique. Arch Franc Pediat 23:5-14

Sheldon W (1964) Congenital pancreatic lipase deficiency. Arch Dis Child 39:268-271

Sims HF, Jennens ML, Lowe ME (1993) The human pancreatic lipase-encoding gene: structure and conservation of an Alu sequence in the lipase gene family. Gene 131:281-285

van Biervliet JP, Vercaemst R, De Keersgieter W, Vinaimont N, Caster H, Rosseneu M (1980) Evolution of lipoprotein patterns in newborns. Acta Paediatr Scand 69:593-596

Yang Y, Sanchez D, Figarella C, Lowe ME (2000) Disco-ordinate expression of pancreatic lipase and two related proteins in the human fetal pancreas. Pediatr Res 47:184-188 